\title{
Tunable plasmonic metasurface for perfect absorption
}

\author{
Paloma Arroyo Huidobro, Stefan A. Maier, and John B. Pendry \\ Imperial College London, Department of Physics, The Blackett Laboratory, London SW7 2AZ, UK
}

Received 6 October 2016 / Accepted 13 January 2017

\begin{abstract}
Tunable metasurfaces, whose functionality can be dynamically modified, open up the possibility of ultracompact photonic components with reconfigurable applications. Here we consider a graphene monolayer subject to a spatially periodic gate bias, which, thank to surface plasmons in the graphene, acts as a tunable and extremely compact metasurface for terahertz radiation. After characterizing its functionality, we show that it serves as the basic building block of an ultrathin complete absorber. In this subwavelength-thickness device, transmission and reflection channels are blocked and electromagnetic energy is completely absorbed by the metasurface building blocks. The proposed structure can be used as a modulator, and its frequency of operation can be changed by scaling its size or adjusting the doping level.
\end{abstract}

Key words: Graphene plasmonics, Tunable metasurface, Perfect absorption.

\section{Introduction}

Metasurfaces, the two-dimensional (2D) counterpart of metamaterials [1, 2], consist of resonant subwavelength-sized building blocks arranged in planar arrays [3]. By appropriately designing the building blocks and their spatial arrangement, metasurfaces provide an ultra-thin platform for controlling electromagnetic (EM) waves [4]. There is currently a great interest in developing reconfigurable metasurfaces, which could be used as tunable ultrathin optical elements. Graphene, with its tunable carrier concentration and 2D character [5], appears as a material with substantial potential for this purpose. Interestingly, metasurfaces may take advantage of the long-lived surface plasmons sustained by graphene at terahertz $(\mathrm{THz})$ frequencies [6-8], which have very short wavelengths and are extremely confined to the sheet [9-17]. This results in a greatly enhanced interaction between graphene and EM radiation, which places graphene as an excellent platform for the design of reconfigurable ultrathin metasurfaces $[18,19]$.

In this work we consider a graphene metasurface based on a monolayer of this $2 \mathrm{D}$ material whose conductivity is spatially modulated on a subwavelength scale, see Figure 1a. By means of analytical expressions derived with the help of transformation optics $[20,21]$, which we demonstrate to be in excellent agreement with full electrodynamic simulations, we characterize the optical spectrum of this metasurface. Next, we utilize this tunable metasurface as building block for the design of a perfect absorber, see Figure $1 \mathrm{~b}$, one of the relevant

*e-mail: p.arroyo-huidobro@imperial.ac.uk functionalities of these ultrathin platforms for modulating EM radiation $[22,23]$.

This manuscript is structured as follows. In Section 2 we consider a plasmonic metasurface composed of a graphene monolayer with spatially modulated conductivity. Next, in Section 3 we design an ultrathin perfect absorber based on the plasmonic metasurface. Finally, in Section 4 we summarise the main conclusions of this work.

\section{Graphene with spatially periodic conductivity: a plasmonic metasurface}

We start by considering a plasmonic metasurface based on a graphene monolayer with spatially periodic conductivity $[24,25]$. The configuration we propose is sketched in Figure 1a. A graphene sheet is placed on top of a substrate (enclosed by permittivies $\varepsilon_{1}$ and $\varepsilon_{2}$ ), and subject to a spatially periodic bias, generated for instance by gating the graphene electrostatically, which can be dynamically controlled. This results in a periodic modulation of the sheet's conductivity, which we assume to be sinusoidal,

$$
\sigma(x)=\sigma_{\mathrm{g}}\left(a_{0}+a_{1} \sin \frac{x}{2 \pi \gamma}\right)
$$

Here, $\sigma_{\mathrm{g}}$ is the conductivity of the homogeneously biased graphene, $2 \pi \gamma$ is the periodicity and $\left\{a_{0}, a_{1}\right\}$ depend on the modulation strength, that we characterize through a single parameter $w_{0}$. In our calculations, we model the conductivity of graphene by a Drude model, which neglects interband contributions and is a good approximation at sufficiently low 


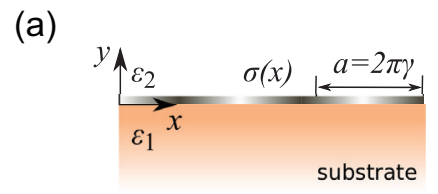

(b)

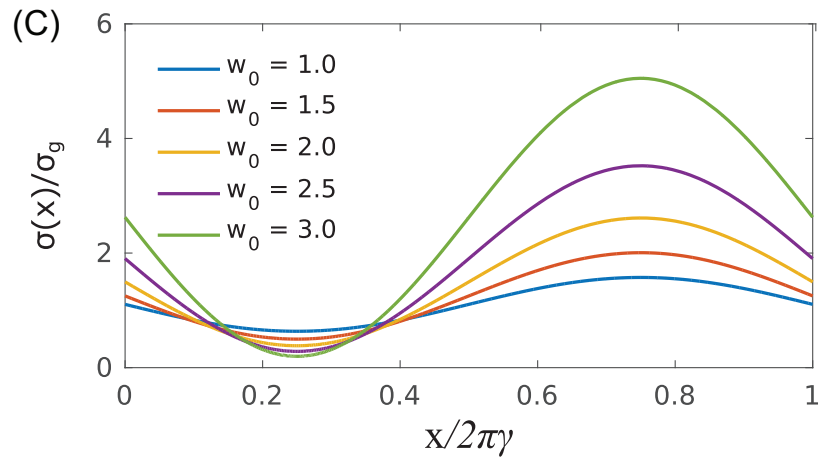

Figure 1. Plasmonic metasurface with spatially modulated graphene. (a) Sketch of the monolayer graphene structure. The conductivity of the graphene sheet is periodically modulated along the $x$ direction, $\sigma(x)$, with period $2 \pi \gamma$. For radiation of wavelengths larger than the periodicity, the graphene appears as a homogeneous sheet with effective conductivity $\sigma_{\text {eff. }}$ (b) Metasurface absorber: the graphene is placed on an asymmetric Fabry-Perot resonator of subwavelength dimensions. (c) Conductivity modulation profile for several values of the modulation strength, $w_{0}$.

frequencies and high doping levels, such as at $\mathrm{THz}$ frequencies. Within this Drude description, the conductivity reads as,

$$
\sigma_{\mathrm{g}}(\omega)=\frac{\alpha}{\gamma_{\mathrm{g}}-\mathrm{i} \omega}
$$

where $\alpha=e^{2} \mu / \pi \hbar^{2}$ is the Drude weight and $\gamma_{\mathrm{g}}=1 / \tau$ is the collision frequency. Here, $\mu$ and $\tau$ are the chemical potential and the scattering time for electrons, respectively, with $\tau=m \mu / e v_{F}^{2} \quad\left(m\right.$ is the mobility and $v_{\mathrm{F}} \approx 10^{6} \mathrm{~m} / \mathrm{s}$ the Fermi velocity). In this work, we use the parameters $\alpha=76 \mathrm{GHz} / \Omega$ and $\gamma_{\mathrm{g}}=1.5 \mathrm{THz}$, which correspond to $\mu=0.65 \mathrm{eV}$ and $m=10^{4} \mathrm{~cm}^{2} / \mathrm{V} \mathrm{s}$.

The conductivity grating established via periodic doping allows for free space radiation to couple into the surface plasmons sustained by the graphene layer, which have much shorter wavelength [24-34]. Hence, the modulation period needs to be much smaller than the wavelength of incident radiation $\left(\lambda_{0} \gg 2 \pi \gamma\right)$. In this regime, the modulated graphene sheet appears as a homogeneous layer for incident radiation, that is, it acts as a metasurface characterized by an effective conductivity.

A graphene sheet with spatially periodic conductivity can be related to a homogeneously biased graphene layer through a conformal transformation (see Refs. [24, 25] for more details). Hence, in the electrostatic limit, both structures have the same plasmon resonance condition $[35,36]$. By applying the transformation optics framework, this analytical mapping allows to derive closed expressions for the reflection and transmission coefficients of the modulated graphene for $p$-polarized radiation at normal incidence,

$$
\begin{aligned}
r_{i j} & =\frac{\sqrt{\varepsilon_{j}}-\sqrt{\varepsilon_{i}}-\eta_{0} \sigma_{\mathrm{eff}}(\omega)}{\sqrt{\varepsilon_{j}}+\sqrt{\varepsilon_{i}}+\eta_{0} \sigma_{\mathrm{eff}}(\omega)}, \\
t_{i j} & =\frac{2 \sqrt{\varepsilon_{j}}}{\sqrt{\varepsilon_{j}}+\sqrt{\varepsilon_{j}}+\eta_{0} \sigma_{\mathrm{eff}}(\omega)} .
\end{aligned}
$$

Here, $\varepsilon_{j}$ and $\varepsilon_{\mathrm{i}}$ are the permittivities of the incidence and transmission sides of the interface, $\eta_{0}=\sqrt{\mu_{0} / \varepsilon_{0}}$ is the impedance of free space and $\sigma_{\text {eff }}(\omega)$ is the effective conductivity of the graphene metasurace. In particular, $\sigma_{\text {eff }}(\omega)=\sigma_{\mathrm{g}}(\omega) N_{\text {TO }}$, where $\sigma_{\mathrm{g}}(\omega)$ is the conductivity of homogeneously biased graphene (Eq. (2)) and $N_{\mathrm{TO}}$ is an analytical factor that contains all the details on geometrical and modulation parameters and dielectric environment, $N_{\mathrm{TO}}\left(\omega, \gamma, w_{0}, \varepsilon_{1}, \varepsilon_{2}\right)$. It can be written in terms of the Fourier expansion coefficients of the conformal transformation and the electrostatic potential expansion (explicit expressions can be found in Refs. [24, 25]). Then, transmittance and reflectance through the modulated graphene are given by $R=|r|^{2}$ and $T_{i j}=\sqrt{\varepsilon_{i} / \varepsilon_{j}}|t|^{2}$. Finally, we note that while the exact shape of the conductivity grating is given by the conformal transformation, the sinusoidal shape we assume is a very good approximation as long as in our calculations the graphene sheet is placed far away from the branch points in the transformed space (see Refs. [24, 25] for more details). On the other hand, the modulation strength $w_{0}$ relates to the parameters $\left\{a_{0}, a_{1}\right\}$ in equation (1) through the conformal transformation. The relation between $w_{0}$ and $\left\{a_{0}, a_{1}\right\}$ is uniquely defined, and both $a_{0}$ and $a_{1}$ increase as $w_{0}$ increases. See Ref. [25] for explicit expressions of their relation. The shape of the conductivity modulation for different values of $w_{0}$ is shown in Figure 1c. It is worth noting that our analytical approach allows us to handle strongly modulated gratings.

Figure 2a shows the reflectance and absorption spectra of the graphene metasurface as a function of modulation strength, $w_{0}$, as calculated from the analytical expressions given by transformation optics (through Eqs. (3) and (4)). The periodicity is $2 \pi \gamma$ with $\gamma=400 \mathrm{~nm}$ and the graphene layer is placed on top of a substrate of refractive index 1.5. Coupling of radiation to the plasmon dipole mode is responsible for the resonant feature appearing both in reflectance (upper panel) and absorption (lower panel). This resonance is at the same frequency $(13 \mathrm{THz})$ for all the values of the modulation strength because all the set of metasurfaces with the same periodicity map into the same homogeneously biased graphene through the conformal transformation. For this reason, the resonance frequency of the plasmon resonance is fixed in all cases by that of a graphene sheet with unmodulated conductivity,

$$
\varepsilon_{1}+\varepsilon_{2}+\frac{4 \pi \sigma_{\mathrm{g}}(\omega)}{\omega}|k|=0,
$$

where $k$ is the plasmon parallel momentum, i.e., the wavevector component along $x$. Hence, given a periodicity, the plasmon resonance is at the same frequency for all gratings. On the other hand, the intensity and width of the resonance peak depend on the modulation strength. The peak in reflectance grows as $w_{0}$ increases, since the 
(a)

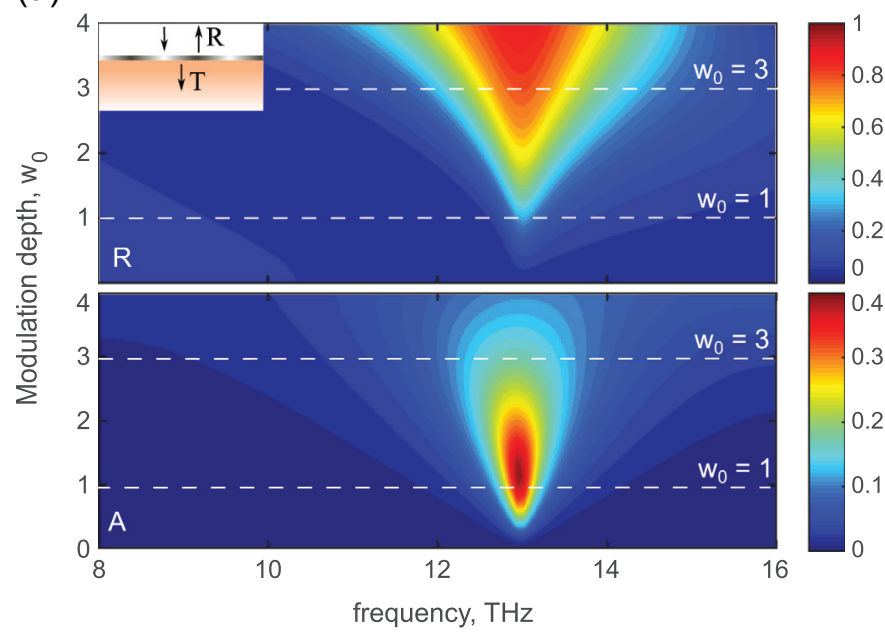

(b)

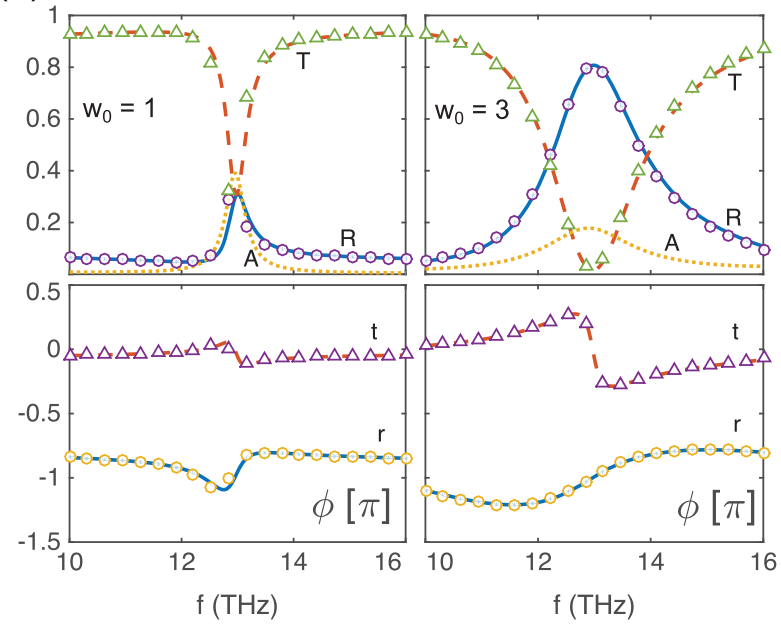

Figure 2. Optical response of the graphene plasmonic metasurface (period $2.5 \mu \mathrm{m}$, permittivities of surrounding media $\varepsilon_{1}=2.25, \varepsilon_{2}=1$ ). (a) Reflectance (upper panel) and absorbance (lower panel) as a function of frequency and strength of the conductivity modulation. (b) Reflectance, absorbance and transmittance spectra (upper panels), as well as relative phase of reflected and transmitted fields (lower panels), for two different values of the modulation strength $\left(w_{0}=1\right.$, left, and $w_{0}=3$, right). For $w_{0}=1, a_{0}$ and $a_{1}$ in equation (1) take the values 1.1048 and 0.4696 , respectively, and for $w_{0}=1, a_{0}=2.6237$ and $a_{1}=2.4225$. The lines show the analytical results calculated with transformation optics, and the dots correspond to full electrodynamic simulations.

coupling of the incident wave to the plasmon mode is larger for stronger modulations. Hence, shallow modulations yield a small increase in reflection at resonance (see the spectrum for $w_{0}=1$ in the upper left panel of Figure 2b), and increasing the modulation strength results in an appreciable increase of reflection. For $w_{0}=3$, reflectance grows to $\sim 80 \%$ (while transmittance approaches 0 , see upper right panel). On the other hand, radiation damping increases with coupling, which results in the absorption spectra reaching a maximum for low values of the modulation strenght, $w_{0} \sim 1$. In addition, the maximum value of absorbance in the metasurface, see for instance Ref. [18], is limited to,

$$
A_{\max }^{i j}=\frac{1}{1+\sqrt{\varepsilon_{i} / \varepsilon_{j}}} .
$$

In this case, $A_{\max }^{12}=0.4$, consistent with the maximum value obtained in the calculations. Finally, the lower panels in Figure $2 b$ show the relative phase of the reflected and transmitted radiation, which take values spanning a range smaller than $\pi$.

\section{Ultrathin perfect absorber}

Here we design an ultrathin metasurface for perfect absorption, formed by combining the already described graphene metasurface with a subwavelength optical cavity, see Figure 1b. A graphene monolayer with effective conductivity $\sigma_{\text {eff }}(\omega)$ is separated by a dielectric spacing layer (thickness $d$, permittivity $\varepsilon_{1}$ ) from an optically thick metal film. For incident radiation, the graphene metasurface acts as a partially reflecting mirror, while the metal at the back acts as a perfectly reflecting mirror. Hence, this structure forms an asymmetric
Fabry-Perot resonator capable of achieving perfect absorption [19, 37-39]. The mechanism underlying complete absorption is the interplay between suppressed reflection owing to destructive interference and on-resonance absorption at the metasurface.

Assuming a plane wave at normal incidence, we start by writing the matching equations for the field amplitudes in each region of space ( $E_{\text {ref }}, E_{\text {inc }}$ and $E^{ \pm}$, see Figure $\left.1 b\right)$, as follows:

$$
\begin{gathered}
E_{\mathrm{ref}}=r_{21} E_{\mathrm{inc}}+t_{12} E^{+}, \\
E^{-}=t_{21} E_{\mathrm{inc}}+r_{12} E^{+}, \\
E^{+}=r_{1 m} E^{-} \exp (2 i k d) .
\end{gathered}
$$

Here, $k$ is the wavenumber in the cavity, $k=2 \pi n_{1} / \lambda_{0}$, and the different reflection coefficients correspond to the $\varepsilon_{2} / \sigma / \varepsilon_{1}$ and $\varepsilon_{1} / \varepsilon_{\mathrm{m}}$ interfaces as the subindexes indicate. In particular, $r_{12}$ and $r_{21}$ correspond to reflection from the graphene metasurface, and have the analytical form given in equation (3), while $r_{1 m}=-1$ (assuming a perfect metal). From this, we can extract the reflection coefficient of the whole system as $r=\frac{E_{\text {ref }}}{E_{\text {ine }}}$,

$$
r=\frac{r_{21}+\left(t_{21} t_{12}-r_{21} r_{12}\right) r_{1 m} \exp (2 i k d)}{1-r_{12} r_{1 m} \exp (2 i k d)},
$$

and reflectance as $R=|r|^{2}$.

Figure 3a presents the reflectance spectrum (left) and the relative phase of reflected radiation (right) as the dielectric spacer thickness, $d$, varies. The graphene metasurface has the same periodicity as in Figure 2, and $w_{0}=3$ was chosen to optimise the structure for a thickness of $1 \mu \mathrm{m}$. The plots show how for this structure reflectance displays resonant minima that 


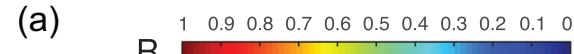
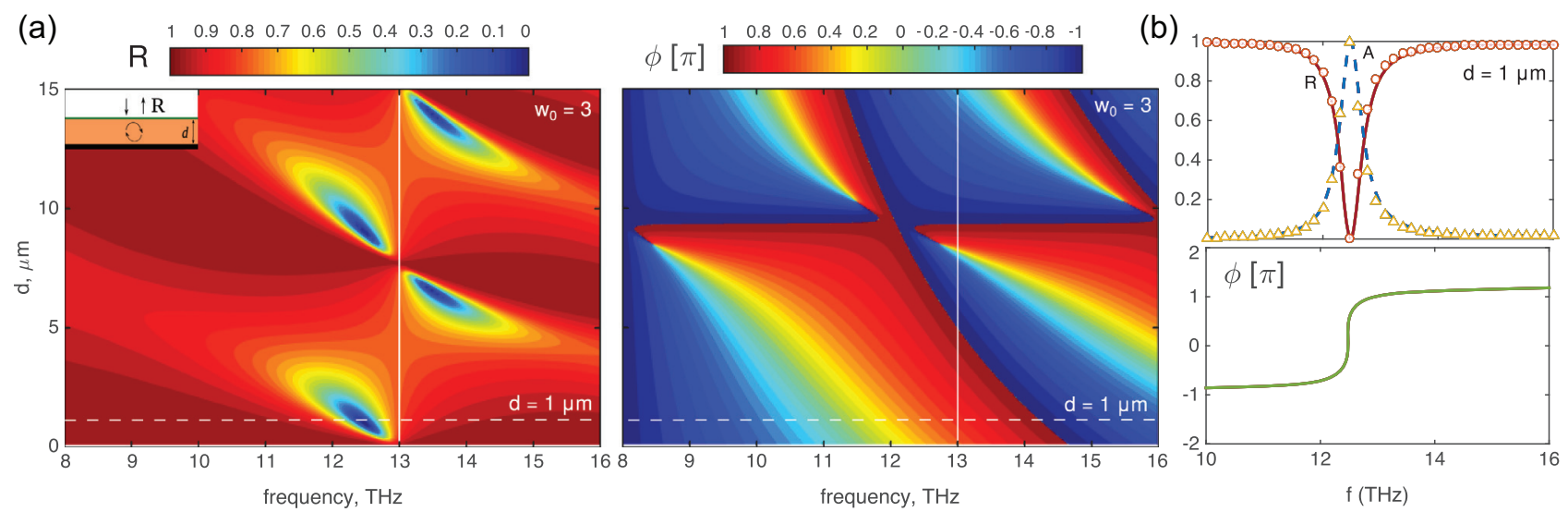

Figure 3. Ultrathin perfect absorber with graphene. Reflectance (a) and relative phase of the reflected field (b) as a function of cavity thickness, $d$. The solid white lines point at the plasmon resonance of the corresponding graphene metasurface without the cavity. (c) Reflectance, absorbance and phase for the case of cavity thickness $d=1 \mu \mathrm{m}$. The lines correspond to analytics and the dots to full electrodynamic simulations. The conductivity modulation strength of graphene was $w_{0}=3$ in all cases.

reach $R=0$, which implies $100 \%$ absorption since the transmission channel is supressed. Such minima are located close to the resonance frequency of the plasmonic metasurface, and periodically displayed as the dielectric layer thickness varies. First, the large coupling between radiation and the plasmonic metasurface results in enhanced absorption close to the plasmon resonance frequency $\sim 13 \mathrm{THz}$. Then, the plasmon mode sustained by the metasurface interacts strongly with the Fabry-Perot resonances in the cavity, giving rise to the anticrossing features visible in the spectrum. Furthermore, this compact structure also provides a phase shift of the reflected wave spanning between $-\pi$ and $\pi$, as opposed to the more limited phase shift observed for the isolated graphene metasurface (see Figure 2b).

The condition for complete absorption in the structure is $r=0$ or, equivalently,

$$
r_{21}+\left(t_{21} t_{12}-r_{21} r_{12}\right) r_{1 m} \exp (2 i k d)=0 .
$$

From this equation it is clear that the phase accumulated within the cavity, $2 k d$, does not have to be close to $\pi$, which makes it possible to achieve the critical coupling condition with a spacing much smaller than the wavelength, $d \ll \lambda$. In addition, note that perfect absorption can be achieved even if the back reflector is not a perfect metal $\left(r_{1 m} \neq-1\right)$, since equation (11) can still be satisfied. The case for a cavity of $d=1 \mu \mathrm{m}$ is shown in panel (b), which displays completely suppressed reflection, $R=0$, thanks to maximum absorption, $A=100 \%$, at $f=12.5 \mathrm{THz}$. This corresponds to a wavelength of incident radiation of $\lambda=24 \mu \mathrm{m}$. Hence, the total thickness of the structure is much smaller than the wavelength of operation, in particular $d=\lambda / 24$. It is important to notice also that the frequency where perfect absorption occurs, $f=12.5 \mathrm{THz}$, is blue-shifted with respect to the surface plasmon condition of the graphene metasurface, $f=13 \mathrm{THz}$. While at the surface plasmon resonance frequency, for $w_{0}=3$, the metasurface reflects most part of the signal and absorption is very low (see Figure 2a), at a blue-shifted frequency the reflection and transmission coefficients are such that the critical coupling condition, equation (11), is exactly satisfied and perfect absorption is achieved.

Finally, we discuss the effect of $\gamma_{\mathrm{g}}$ in the bandwidth of operation. Losses in the graphene directly control the width of the plasmon resonances in modulated graphene: lower $\gamma_{\mathrm{g}}$ implies sharper resonances. On the other hand, while decreasing $\gamma_{\mathrm{g}}$ also reduces the operation bandwidth of the metasurface absorber, it also has an effect on the position of the minimum absorbance resonance. This is because changing the conductivity of graphene affects the critical coupling condition through the reflection and transmission coefficients of the modulated graphene (Eq. (11)). As a general rule, decreasing $\gamma_{\mathrm{g}}$ moves the perfect absorption minimum to smaller values of $d$ as the bandwidth is reduced.

\section{Conclusions}

To summarize, we have considered a plasmonic metasurface based on a graphene monolayer subject to a periodically modulated doping. By means of analytical expressions for the normal incidence reflection and transmission coefficients through a graphene sheet with a spatially modulated conductivity with a sinusoidal profile, we have characterized the performance of this metasurface showing strong resonant features due to efficient coupling to surface plasmons.

In the second part of this work, we have studied an ultrathin perfect absorber, capable of reaching 100\% absorption while being much thinner than the wavelength of radiation, $d=\lambda / 24$. This is achieved by coupling the graphene metasurface to a subwavelength optical cavity, which allows for minimizing reflection when the critical coupling condition is achieved. Since the transmission channel is suppressed, total absorption is achieved. Hence, this ultrathin structure serves as a modulator operating at $\mathrm{THz}$ frequencies with modulation depths approaching $100 \%$, which could be modulated at a speed of up to tens of GHzs [38]. Furthermore, this structure has the potential of rendering omnidirectional perfect absorption by using a graphene metasurface with periodically 
modulated conductivity along two directions [25], which presents an isotropic response to incident radiation.

Acknowledgements. This work was supported by the Leverhulme Trust, the EPSRC (Grant No. EP/L024926/1), and the Gordon and Betty Moore Foundation. P.A.H. acknowledges afunding from a Marie Sklodowska-Curie Fellowship. S.A.M. additionally acknowledges the Lee-Lucas Chair in Physics.

\section{References}

1. D.R. Smith, J.B. Pendry, M.C.K. Wiltshire, Science (New York, NY) 305 (2004) 788.

2. C.M. Soukoulis, M. Wegener, Nat. Photonics 5 (2011) 523.

3. A.V. Kildishev, A. Boltasseva, V.M. Shalaev, Science 339 (2013) 1232009.

4. M. Khorasaninejad, W.T. Chen, R.C. Devlin, J. Oh, A.Y. Zhu, and F. Capasso, Science 352, 1190 (2016), http://science. sciencemag.org/content/352/6290/1190.full.pdf

5. F. Bonaccorso, Z. Sun, T. Hasan, A.C. Ferrari, Nat. Photonics 4 (2010) 611.

6. L. Ju, B. Geng, J. Horng, C. Girit, M. Martin, Z. Hao, H.A. Bechtel, X. Liang, A. Zettl, Y.R. Shen, F. Wang, Nat. Nanotechnol. 6 (2011) 630.

7. P. Tassin, T. Koschny, C.M. Soukoulis, Science 341 (2013) 620.

8. T. Low, P. Avouris, ACS Nano 8 (2014) 1086.

9. O. Vafek, Phys. Rev. Lett. 97 (2006) 266406.

10. G.W. Hanson, J. Appl. Phys. 103 (2008) 064302.

11. M. Jablan, H. Buljan, M. Soljačić, Phys. Rev. B: Condens. Matter Mater. Phys. 80 (2009) 245435.

12. A.A. Dubinov, V.Y. Aleshkin, V. Mitin, T. Otsuji, V. Ryzhii, J. Phys.: Condens. Matter 23 (2011) 145302.

13. F.H.L. Koppens, D.E. Chang, F.J. García de Abajo, Nano Lett. 11 (2011) 3370.

14. A.Y. Nikitin, F. Guinea, F.J. García-Vidal, L. Martín-Moreno, Phys. Rev. B: Condens. Matter Mater. Phys. 84 (2011) 195446.

15. Z. Fei, G.O. Andreev, W. Bao, L.M. Zhang, A.S. McLeod, C. Wang, M.K. Stewart, Z. Zhao, G. Dominguez, M. Thiemens, M.M. Fogler, M.J. Tauber, A.H. Castro-Neto, C.N. Lau, F. Keilmann, D.N. Basov, Nano Lett. 11 (2011) 4701.

16. Z. Fei, A.S. Rodin, G.O. Andreev, W. Bao, A.S. McLeod, M. Wagner, L.M. Zhang, Z. Zhao, M. Thiemens, G. Dominguez, M.M. Fogler, A.H. Castro-Neto, C.N. Lau, F. Keilmann, D.N. Basov, Nature 487 (2012) 82.

17. J. Chen, M. Badioli, P. Alonso-González, S. Thongrattanasiri, F. Huth, J. Osmond, M. Spasenović, A. Centeno, A. Pesquera, P. Godignon, A.Z. Elorza, N. Camara, F.J. García de Abajo, R. Hillenbrand, F.H.L. Koppens, Nature 487 (2012) 77.
18. Y. Fan, N.-H. Shen, T. Koschny, C.M. Soukoulis, ACS Photonics 2 (2015) 151.

19. Z. Li, K. Yao, F. Xia, S. Shen, J. Tian, Y. Liu, Sci. Rep. 5 (2015) 12423.

20. A.J. Ward, J.B. Pendry, J. Modern Optics 43 (1996) 773.

21. J.B. Pendry, D. Schurig, D.R. Smith, Science (New York, NY) 312 (2006) 1780.

22. N.I. Landy, S. Sajuyigbe, J.J. Mock, D.R. Smith, W.J. Padilla, Phys. Rev. Lett. 100 (2008) 207402.

23. T. Teperik, F. García de Abajo, A. Borisov, M. Abdelsalam, P. Bartlett, Y. Sugawara, J. Baumberg, Nature Photonics 2 (2008) 299.

24. P.A. Huidobro, M. Kraft, R. Kun, S.A. Maier, J.B. Pendry, J. Opt. 18 (2016) 44024.

25. P.A. Huidobro, M. Kraft, S.A. Maier, J.B. Pendry, ACS Nano 10 (2016) 5499

26. Y.V. Bludov, N.M.R. Peres, M.I. Vasilevskiy, Phys. Rev. B: Condens. Matter Mater. Phys. 85 (2012) 245409.

27. T.R. Zhan, F.Y. Zhao, X.H. Hu, X.H. Liu, J. Zi, Phys. Rev. B: Condens. Matter Mater. Phys. 86 (2012) 165416.

28. A.Y. Nikitin, F. Guinea, F.J. Garcia-Vidal, L. Martin-Moreno, Phys. Rev. B: Condens. Matter Mater. Phys. 85 (2012) 081405 (R).

29. A.Y. Nikitin, F. Guinea, L. Martin-Moreno, Appl. Phys. Lett. 101 (2012) 151119.

30. S. Thongrattanasiri, F.H.L. Koppens, F.J. García De Abajo, Phys. Rev. Lett. 108 (2012) 047401.

31. E. Forati, G.W. Hanson, A.B. Yakovlev, A. Alù, Phys. Rev. B: Condens. Matter Mater. Phys. 89 (2014) 1.

32. P. Alonso-González, A.Y. Nikitin, F. Golmar, A. Centeno, A. Pesquera, S. Vélez, J. Chen, G. Navickaite, F. Koppens, A. Zurutuza, F. Casanova, L.E. Hueso, R. Hillenbrand, Science (New York, NY) 344 (2014) 1369.

33. T. Stauber, G. Gómez-Santos, and F. J. De Abajo, Phys. Rev. Lett. 112 (2014) 077401.

34. Z. Miao, Q. Wu, X. Li, Q. He, K. Ding, Z. An, Y. Zhang, L. Zhou, Phys. Rev. X 5 (2015) 041027.

35. M. Kraft, J.B. Pendry, S.A. Maier, Y. Luo, Phys. Rev. B 89 (2014) 245125.

36. M. Kraft, Y. Luo, S.A. Maier, J.B. Pendry, Phys. Rev. X 5 (2015) 031029.

37. R. Alaee, M. Farhat, C. Rockstuhl, F. Lederer, Opt. Express 20 (2012) 28017.

38. Y. Yao, R. Shankar, M.A. Kats, Y. Song, J. Kong, M. Loncar, F. Capasso, Electrically tunable metasurface perfect absorbers for ultrathin mid-infrared optical modulators, Nano Letters 1411 (2014) 6526-6532.

39. M.S. Jang, V.W. Brar, M.C. Sherrott, J.J. Lopez, L. Kim, S. Kim, M. Choi, H.A. Atwater, Phys. Rev. B 90 (2014) 165409. 